LIPIDS

\section{Protomembranes}

from our Molecular Biology Correspondent

IT seems that the study of hydrocarbons will for some time continue to provide a comfortable livelihood for many a physical chemist in the guise of a molecular biologist. The possibilities in liposomes are still far from exhausted, and McConnell especially has shown how much structural and dynamic information can be extracted from his spin-label experiments on such systems.

Cadenhead and Katti (Biochim. Biophys. Acta, 241, 709; 1971) have tried to step on the cake by showing that a molecule carrying a large and ugly spin-label group will not necessarily have the same physical properties as the virgin form. There is no complete counter to this criticism, though it can be argued that inasmuch as spinlabelled lipids can be biosynthetically incorporated in membranes, they seem tc. be at least compatible, if not identical in behaviour, with normal lipids. Moreover the results of other methods, including NMR and X-ray diffraction, seem to be converging on much the same conclusions. Cadenhead and Katti, however, have looked into the effects of oxyloxazolidine-substituted cholesterol-like steroids on the surface properties of films, in which they are mixed with myristic acid. The polar spin-label group, they find, changes the effect on the surface isotherms considerably, compared with the parent compound; they suggest that this will generally be the case and recommend caution in visiting the results of spinlabelled onto unlabelled systems.

At the same time Birdsall et al. (ibid., 693) offer support for the conclusions of Hubbell and McConnell concerning configurational freedom of lipid chains in bilayers. Instead of introducing the relatively large and polar spin-label, they have prepared a series of stearic acids containing a fluorine atom at various positions in the paraffin chain. These derivatives were then mixed with lecithin and dispersed in water as vesicies. NMR signals from the ${ }^{19} \mathrm{~F}$ nucleus revealed that the line-widths changed drastically with the distance of the fluorine from the head group, indicating a large increase in freedom of motion going from the outside to the centre of the bilayer. This is by now a heavily laboured conclusion. It may be hoped, however, that a more complete analysis, including an estimate of exchange line-broadening effects, will make possible a quantitative treatment, so as to allow quantitative comparison with the results of other methods.

The most interesting current development in the application of NMR to the study of such systems comes from the Moscow group (Bystrov et al., Chem. Phys. Lipids, 6, 345; 1971), who have examined the effect of paramagnetic perturbants on the proton magnetic resonance spectra of lecithin vesicles. When vesicles are prepared in water (or actually $\mathrm{D}_{2} \mathrm{O}$ ) containing manganous ions, there is a spectacular broadening effect, primarily of the signal from the quaternary amine methyl hydrogens, because these are in contact with the aqueous medium on the inside and outside of the vesicle. The ions do not penetrate the bilayer, however, so that when the vesicles are made in water and the paramagnetic ions are only afterwards added, a quite different effect is observed: superimposed on the shallow broadened background is a sharp peak of diminished magnitude. The polar heads on the inner surface are not in contact with the ions, and thus give rise to the sharp unperturbed resonance, whereas those on the outer surface generate only the broadened pedestal. When such preparations are sonicated, the residual sharp line from the internal head groups vanishes into the broadened background. An equally spectacular effect is observed with europium ions, which elicit a large contact-shift in the head-group reson- ance, so that when they are added to preformed vesicles, two sharp signals, one from the internal, the other from the external head groups, are observed.

Bystrov et al. also prepared micelles of lecithin in benzene, and found that these could be made to take up water. Again the introduction of the paramagnetic perturbent perturbed only the head groups exposed to water on the inner surface. These experiments offer good promise for the separate observation of events at different surfaces both of models and of real cell membranes.

A review by Kuhn and Möbius (Angew. Chem., 10,620;1971) of their remarkable work on monolayer systems, and the use of excitation transfer to measure critical distances, will give workers in the biological membrane field much food for rumination. The scope of this work takes in many areas of chemistry, but some examples of biological interest have been included in the survey, in particular the measurement of membrane thickness by introducing the membrane between emitter and sensitive layers and measuring the efficiency of excitation transfer and the construction to order of various arrangements of lipid and protein monolayers.

\title{
Rescuing Mouse Sarcoma Virus
}

IN next Wednesday's Nature New Biology, Klement, Nicolson and Huebner report that they have rescued mouse sarcoma virus particles from rat cells which although transformed by this virus do not support its replication until they are exposed to appropriate concentrations of 5-bromodeoxyuridine (BUdR).

This discovery, which follows hard on the heels of those of Rowe's group (Science, 174, 155; 1971) and Aaronson, Todaro and Scolnick (ibid., 157), who have used $B U d R$ and $I U d R$ to rescue respectively mouse leukaemia virus from non-producing AKR strain mouse embryo cells and a C-type virus, of which the oncogenic potential has yet to be tested, from normal BALB/C 3T3 fibroblasts, is a powerful shot in the arm for the devotees of the oncogene hypothesis of malignant transformation. And of course the induction with BUdR of IUdR technique provides a new approach to the search for other mammalian, including human, RNA tumour viruses.

The expcriments of the three groups, which are essentially identical, indicate that the drugs BUdR and IUdR, for reasons that are far from clear at present, stimulate the active replication of dormant RNA tumour virus genomes in rat and mouse cells. The virus which
Rowe's group have activated in embryonic cells of the AKR strain, a high leukaemia strain, seems to be identical to that isolatable from adult AKR mice with the disease. The virus which Todaro's group have activated in 3T3 cells has all the vital statistics of a Ctype RNA tumour virus and it will be fascinating to learn whether or not it is indeed oncogenic. And Huebner's group believe that the rescue of mouse sarcoma virus from transformed but non-producing rat cells is most probably a consequence of the activation of a dormant rat "leukaemia" virus which can provide the helper function required for the replication of mouse sarcoma virus and normally provided by mouse leukaemia virus.

The avian oncogenic virologists, and Weiss in particular, have, of course, already shown that a C-type virus, probably an avian leukosis virus, can be activated in the cells of domestic chicks and wild jungle fowl by various chemicals including carcinogens, and so the betting must be that all vertebrate cells have the genetic potential to specify C-type viruses at least some of which are oncogenic. And that is what Huebner and Todaro's oncogene hypothesis predicts; perhaps all cells do indeed carry genes which can render them malignant. 